\title{
MIR-361-5P Mediates TGF- $\beta$ Signalling to Promote Granulosa Cell Apoptosis Through Vegfa During Porcine Follicle Atresia
}

\section{Mengnan Ma}

Nanjing Agricultural University

Xiaomeng Gao

Nanjing Agricultural University

Wang Yao

Nanjing Agricultural University

Zengxiang Pan

Nanjing Agricultural University

jinbi zhang ( $\nabla$ zhangjinbi@njau.edu.cn )

Nanjing Agricultural University https://orcid.org/0000-0002-9492-3425

\section{Research}

Keywords: Follicular atresia, GC apoptosis,VEGFA,miR-361-5p, TGF- $\beta$ signalling, SMAD4

Posted Date: June 13th, 2020

DOI: https://doi.org/10.21203/rs.3.rs-34712/v1

License: (c) (1) This work is licensed under a Creative Commons Attribution 4.0 International License. Read Full License 


\section{Abstract}

Background: Follicular atresia is an inevitable degenerative process of ovarian follicles in mammals. The molecular events involved in atresia, particularly in granulosa cell apoptosis, have always attracted researchers' attention. It is known that VEGFA isdownregulated during follicular atresia in pig ovaries and serves as an apoptosis inhibitor of granulosa cells. Besides, the TGF- $\beta$ signalling has been considered as a central trigger in granulosa cell apoptosis. However, the link between TGF- $\beta$ signalling and VEGFA is missing.

Results:We proved that miR-361-5p significantly up-regulated during the atresia process andenhances GC apoptosis by direct targeting to VEGFA 3'UTR. In addition, we revealed that miR-361-5p coding geneMIR361was significant downregulation bySMAD4, the central intracellular mediator of TGF- $\beta$ signalling,through promoter binding.

Conclusions: Our findingsenriched the knowledge of VEGFA post-transcriptional regulation and completed the TGF- $\beta$ /miR-361-5p/VEGFAregulatory networkin ovarian granulosa cell apoptosis. It offereduseful references for follicular atresia and ovarian physiological function studies.

\section{Background}

Follicular atresia is a common physiological phenomenon, which may happen at all stages of follicular development in mammals. In porcine primordial follicle reserve at puberty, there are approximately 5 million primordial follicles, most of which go through the atresia process when they passed the size of $1 \mathrm{~mm}$ of diameter during the antral stage. The atresia rate remarkably increases in antral follicles around 3-5 mm in diameter (Marchal et al., 2002). In the end, the majority of follicles disappear before maturation, and less than $14 \%$ can reach ovulation (Manabe et al., 2004).

Adequate blood flow is necessary for oxygen and nutrient supply in the ovary and possibly a rate-limiting step in the selection and maturation of dominant follicles destined for ovulation (Stouffer et al., 2001). It is known that the vascular endothelial growth factor (VEGF) family, which is composed of at least six members (VEGF A-F), is involved in the formation of blood vessels. VEGFA is the first identified and mostly studied molecule, which is primarily known as a promoting factor of angiogenesis and vasculogenesis (López et al., 2017). It is considered that the function of VEGFA is achieved through its major tyrosine kinase receptor VEGFR2 (also known as kinase insert domain receptor, KDR) (Greenaway et al., 2004). The expression and function of VEGFA have been studied in mammal ovaries during follicular development. In human, VEGF mRNA and protein presented in theca layer and granulosa cells (GCs) during antral follicle stages, when vascular network developed actively (Yamamoto et al., 1997); In bovine, VEGFA mRNA also expressed in both theca interna and GCs, while the VEGF protein level increased with developmental stages of follicle growth (Berisha et al., 2000); In pig, expression levels of two VEGFA isoforms (VEGF120 and VEGF164) in GCs and levels of two receptors (VEGFR1 and VEGFR2) in theca cells appeared to be higher in medium and large follicles than in small ones (Shimizu et al., 
2002). According to our earlier study in pig, VEGFA mRNA level significantly decreased in early atretic follicles compare to the healthy ones (Zhang et al., 2018a), which implied a potential role of VEGFA during follicular atresia.

When it comes to the transcriptional regulation of VEGFA, transcriptional factors including hypoxiainducible factor (HIF) (Damert et al., 1997), estrogen receptors $\alpha$ and $\beta$ (ER a, $\beta$ ) (Buteaulozano et al., 2002), signal transducer and activator of transcription 3 (STAT-3), and Wilms Tumor 1 (WT1) (Hanson et al., 2007) were identified to regulate VEGFA through direct promoter binding. In recent years, more and more studies have focused on the negative post-transcriptional regulation of VEGFA through miRNAs. Direct binding microRNAs such as miR-26a (Chai et al., 2013), miR-93 (Jianyin Long, 2010), miR-134 (Zhang et al., 2018b), miR-195 (Zhao et al., 2017), miR-203 (Zhu et al., 2013), miR-361-5p (Alexander et al., 2012) and miR-503 (Zhou et al., 2013) on VEGFA 3'UTR were identified in a variety of cells and conditions, mostly in carcinoma. However, despite its key function in the ovary, the post-transcriptional regulation of VEGFA in ovarian follicles, especially during atresia, is still unknown.

It has been reported that the canonical transforming growth factor (TGF)- $\beta$ signalling pathway is involved in the proliferation and apoptosis of porcine ovarian granulosa cells. In our previous studies, SMAD family member 4 (SMAD4), the terminal regulatory molecule in TGF- $\beta$ signalling, was identified as an anti-apoptosis factor in granulosa cells (Zhang et al., 2010). Interestingly, this function of SMAD4 was related to its direct binding to the promoter of a few miRNA coding genes and negative regulation of miRNA expression (Xing et al., 2016), which implied a possible regulatory axis of SMAD4-miRNAsfunctional genes in GCs. Thus, we performed the study to identify a potential regulatory axis of SMAD4/miR-361-5p/VEGFA in porcine GCs. The study not only filled the gap of miRNA regulated VEGFA during follicular atresia but also provided evidence for the anti-apoptosis role of SMAD4 through transcriptional regulation of miRNAs in GCs.

\section{Methods}

\subsection{Follicle Collection}

Ovaries were obtained from mature sows at a local slaughterhouse. Individual antral follicles, approximately 3 to $5 \mathrm{~mm}$ in diameter, were dissected from the ovaries after quickly washing twice with $75 \%$ ethanol and physiologic saline using small scissors and fine forceps and then classified into healthy follicles (HFs) and atretic follicles (AFs) according to follicle shape, GC density, and hormone levels (Zhang et al., 2018a).

\subsection{Cell Culture and Transfection}

Primary GCs were obtained from HFs by syringing with a 20-gauge needle and cultured as previously described (Liu et al., 2018). HEK293 cells were cultured as previously described (Liu et al., 2018). VEGFA siRNA, Smad4 siRNA, miR-361-5p mimic, and miR-361-5p inhibitor were synthesized by GenePharma (Shanghai, China) (Supplementary Table S1). After $12 \mathrm{~h}$ of culture, the porcine GCs were transfected with 
the appropriate plasmids or oligos using Lipofectamine 2000 and Opti-MEM (Invitrogen, Carlsbad, CA, USA) according to the manufacturer's protocol.

\subsection{Immunohistochemical assay}

To examine the expression and location of VEGFA in healthy and atretic follicles, immunohistochemical staining was performed according to our previous description(Gao et al., 2020). Rabbit polyclonal VEGFA (diluted 1: 200 in PBS containing1\% (W/V) bovine serum albumin, ab9570, Abcam, Cambridge, MA, USA) and secondary antibody (G1210-2-A, Servicebio, Wuhan, China) was applied. The specific protein immunoreactivity was visualized with $0.05 \%$ 3,30-diaminobenzidine (DAB, G1211, Servicebio, Wuhan, China) for $15 \mathrm{~min}$, and the slides were counterstained with hematoxylin (G1004, Servicebio, Wuhan, China). The images were captured under the microscope (Nikon Eclipse E200, Tokyo, Japan).

\subsection{RNA extraction and qRT-PCR}

Total RNA was extracted from the follicles and GCs using Trizol reagent (Invitrogen, Carlsbad, CA, USA). Then the extracted total RNA was reverse transcribed to cDNA by Super M-MLV RTase Synthesis Kit and qRT-PCR was performed using SYBR Premix Ex Taq (Takara, Dalian, China) on the ABI StepOne system (Applied Biosystems, Carlsbad, CA, USA) according to the manufacturer's instructions. GAPDH was used as an internal control. For miRNA detection,frst-strand cDNA synthesis and qRT-PCR were performed using miRNA two-step qRT-PCR SuperMix (TransGen, Beijing, China). U6 was used as internal control. For each gene, controls for each primer set containing no cDNA were included on each plate, and the reaction was repeated three times for every sample. The primers for qRT-PCR are listed in Supplementary Table S2.

\section{5 fluorescent in situ hybridization (FISH)}

The FAM-labeled probe (5'-GTACCCCTGGAGATTCTGATAA-3 ') was specifically synthesized for miR-361$5 p$, and DAPI was used to stain the cell nuclei. The GCs were cultured on coverslips, fixed in $4 \%$ paraformaldehyde (containing DEPC) for 20 min, washed with shaking in PBS (PH7.4) for three times, and proteinase $\mathrm{K}(20 \mathrm{ug} / \mathrm{ml})$ was finally added for $5 \mathrm{~min}$ for digestion. Then all the procedures were conducted according to the manufactory's instruction (Sevicebio, Wuhan, China). Finally, the images were acquired on a Nikon upright fluorescence microscope (Nikon DS-U3, Japan). Each experiment was performed three times.

\subsection{Protein extraction and western immunoblotting analysis}

GCs were washed with cold PBS and lysed with RIPA buffer containing $1 \%$ phosphatase inhibitor $(v / v)$ (Beyotime, Shanghai, China) and proteinase inhibitor (Sigma, St. Louis, MO, USA). The protein concentration was determined by the BCA Protein Assay Kit (Beyotime, Shanghai, China) and diluted to the same concentration using the $5 \times$ Protein Loading Dye (Sangon, Shanghai, China). Total protein extracts were separated using SDS-PAGE on $12 \%$ gels. The proteins were then transferred onto the PVDF membranes (Millipore, Billerica, MA, USA), and the membranes were blocked with $5 \%$ non-fat milk for $2 \mathrm{~h}$. After washing with Tris-buffered Saline with Tween (TBST) for $15 \mathrm{~s}$, the membranes were incubated with 
anti-VEGFA (diluted 1: 5000, ab9570, Abcam, Cambridge, MA, USA), anti-Tubulin (diluted 1:1000, 10094-1AP ProteinTech, Nanjing, China), anti-caspase3 (diluted 1:1000, 19677-1-AP, ProteinTech, Nanjing, China) overnight at $4{ }^{\circ} \mathrm{C}$. Then incubated with a secondary peroxidase-conjugated antibody (diluted 1:2000, Cell Signaling Technology, Beverly, MA, USA) for $1 \mathrm{~h}$ at room temperature. Chemiluminescence was detected by WesternBright ${ }^{\text {TM }}$ ECL (Advansta, Menlo Park, CA USA) and analyzed using Image $J$ software. Each experiment was performed three times.

\subsection{Plasmid construction}

The 3'-UTR fragments of VEGFA containing putative target sites of miR-361-5p and the promoter fragments of the miR-361-5p coding gene (MIR361) containing putative SMAD4 binding sites were amplified from porcine genomic DNA and verified by sequencing. The VEGFA 3'-UTR fragment was digestion with Nhel and Xbal (Thermo, Waltham, MA, USA), and then cloned into pmirGLO Dualluciferase miRNA Target Expression Vector (Promega Corporation, Madison, WI, USA). The MIR361 promoter fragment was digestion with Nhel and Sacl and then cloned into pGL-3 reporter vector (Promega, USA). The mutant plasmids of the miR-361-5p putative binding site were generated by ClonExpress Entry One Step Cloning Kit (Vazyme, Nanjing, China) according to the manufacturer's protocol. The successful mutations were identified by sequencing technology. The overexpression plasmid pcDNA3.1-SMAD4 plasmid was generated previously by our group (Du et al., 2018). Primers used here are detailed in Supplementary Table S3.

\subsection{Luciferase Reporter Assays}

After a transfection period of $24 \mathrm{~h}$, the cells and lysates were collected. A Dual-Luciferase Reporter Assay System (Promega Corporation, Madison, WI, USA) was used to quantify luciferase activities following the manufacturer's instructions. Firefly luciferase activity was normalized to Renilla luciferase activity. Each experiment was performed six times.

\subsection{Apoptosis assay}

GCs apoptosis was measured by annexin V-FITC/PI staining assay (Vazyme, Nanjing, China) according to the manufacturer's protocol. A cell-counting machine (Becton Dickinson, USA) was used for the detection of apoptotic cells based on the principle of fluorescence-activated cell sorting (FACS). The data were analyzed using FlowJo v7.6 software (Stanford University, Stanford, CA, USA).

\subsection{Statistical analysis}

All data are presented as means \pm S.E.M. Prism 5 software (GraphPad Software) was used to perform statistical analysis. Two-tailed Student's t-test was used to evaluate the significance when two groups were compared. When three or more groups were compared, a one-way analysis of variance test was performed, and Turkey's test to determine significance between groups. P-value of $<0.05$ and 0.01 was considered significant and extremely significant differences, respectively.

\section{Results}




\subsection{VEGFA is downregulated in atretic follicles}

To investigate the VEGFA level during follicular atresia, we first determined the location of VEGFA in antral follicles by immunohistochemistry. The results showed that a positive reaction was observed in both theca and granulosa cells, and the brown staining was stronger in HFs (GCs closely arranged) than in AFs (GCs loosely arranged and partially dropped into the follicular cavity) (Fig. 1.A-D). In addition, mRNA levels of VEGFA detected in the whole follicle, GC, and theca cell (TC) by GeneChip Porcine GenomeArray (detailed data was contained in reference (Zhang et al., 2018a)) or qRT-PCR also suggested a significantly higher expression in HFs compare to atretic ones (Fig. 1.E-G). The ELISA detection of VEGFA showed a slight but significant decrease of VEGFA content in follicular fluid during the atresia process (Fig. 1.H). These results suggested that VEGFA decreases during porcine follicular atresia.

\section{2 miR-361-5p is up-regulated in atretic follicles}

To investigate the possible function of miR-361-5p during follicle atresia, we detected its location and expression levels in healthy and atretic follicles. FISH results showed that miR-361-5p distributed in both TCs and GCs, and the signal was stronger in atretic follicles compare to the healthy ones (Fig. 2.A-F). Further qualitative measurement in the whole follicle, GC, TC, and follicular fluid by $\mu$ Paraflo ${ }^{T M}$ microfluidic chip (detailed data was contained in reference (Lin et al., 2012)) or qRT-PCR, respectively, also suggested a significantly higher expression of mir-361-5p in atretic follicles compare to healthy ones in each component of the follicle (Fig. 2.G-J). These results implied that miR-361-5p was involved in the atresia process and may involve in post-transcriptional regulation of functional genes during follicular atresia.

\section{3 miR-361-5p regulates VEGFA by direct 3'UTR binding}

To further investigate the possible function of miR-361-5p in the regulation of VEGFA expression, the direct targeting of VEGFA by miR-361-5p was first predicted by bioinformatic methods and confirmed by luciferase reporter assay (Fig. 3.A). Next, we cultured porcine GCs, transfected with miR-361-5p mimics or inhibitor, and then detected the mRNA/protein levels of VEGFA in GCs and VEGFA secretion in culture media. The results showed that both mRNA (Fig. 3.B, C) and protein (Fig. 3.D, E) levels of VEGFA were significantly decreased after transfection of miR-361-5p mimics, while increased after transfection of miR-361-5p inhibitor (Fig. 3.B-E). Besides, we noticed that VEGFA secretion in culture media was slightly increased after transfection of miR-361-5p inhibitor (Fig. 3.F, G). These results suggested that miR-361-5p negatively affected VEGFA expression by direct binding to its $3^{\prime} U T R$ in porcine GCs.

\section{4 miR-361-5p regulates GC apoptosis through VEGFA}

To reveal whether miR-361-5p affects apoptosis of porcine GCs via regulation of VEGFA, we cotransfected miR-361-5p inhibitor with VEGFA siRNA. The FACS result suggested that the apoptosis rate was significantly dropped after miR-361-5p inhibitor transfection, but reversed after additional VEGFA 
siRNA (Fig. 4.A). The protein levels of active cleaved Caspase 3 (c-CAS3) also showed a similar pattern (Fig. 4.B). Thus, it is clear that miR-361-5p could promote GC apoptosis through VEGFA.

\subsection{SMAD4 involved in miR-361-5p mediated VEGFA expression}

To examine whether the expression of miR-361-5p was under the control of TGF- $\beta$ signalling pathway, we predicted the promoter region of the miR-361-5p coding gene, MIR361. We identified 4 SMAD-binding elements (SBEs) within the region (Fig. 5.A). Next, we confirmed the promoter activity of the MIR361 promoter region with SBEs using a dual-luciferase reporter assay (Fig. 5.B). To further investigate the effect of SMAD4 on MIR361 promoter, the increase and knockdown of SMAD4 were achieved by SMAD4 overexpression plasmid and siRNA, respectively. The luciferase reporter assays suggested that SMAD4 had a negative effect on the MIR361 promoter (Fig. 5.C, D). In addition, in cultured porcine GCs, overexpression of SMAD4 did not show an apparent effect on miR-361-5p, but knockdown of SMAD4 significantly enhanced miR-361-5p expression (Fig. 5. E, F). Finally, both qRT-PCR and WB suggested that knockdown of SMAD4 resulted in a significant decrease of VEGFA expression (Fig. 5. G, H). These results indicated that SMAD4 regulated MIR361 transcription negatively as a trans-acting element and thereby adjusted VEGFA expression.

\section{Discussion}

miRNAs regulate gene expression by binding to specific sequences on target mRNAs and results in transcriptional repression (Wahid et al., 2010) or degradation (Meister et al., 2004) of target mRNAs. In the field of reproduction, the critical roles of miRNAs in ovarian function, follicle development, and luteal formation have attracted constant attention and were recently connect to GC apoptosis and the follicular atresia processes in human, mouse, bovine and pig (Zhang et al., 2019). Our previous study, which compared the differential expression of miRNAs between healthy and atretic follicles, has suggested a significant raise of miR-361-5p during atresia (Lin et al., 2012). The fact that miR-361-5p inhibited cell proliferation, metabolism, and induce cell apoptosis in many cancer studies (Ma et al.; Ma et al., 2015; Liu et al., 2019) also implied a possible relationship between miR-361-5p and GC apoptosis during atresia process. In this study, we confirmed a direct interaction between miR-361-5p and VEGFA, which was mainly produced in GCs and play essential roles in angiogenesis, GCs function and oocyte development in antral follicles(Gao et al., 2020). In addition, our study explored the transcriptional regulation of the miR-361-5p coding gene, MIR361, by SMAD4 and thus identified a straightforward regulatory network of SMAD4/miR-361-5p/VEGFA. Briefly, miR-361-5p reduces VEGFA mRNA expression by direct binding, thus promotes GC apoptosis, while SMAD4 increases VEGFA level through negative regulation of MIR361 expression through promoter binding at the transcription level (Fig. 6).

It is known that SMAD4 is the universal mediator that plays a role in canonical TGF- $\beta$ signal transduction into the nucleus, where the SMAD complexes regulate gene transcription positively or negatively with different coactivator or corepressor factors (Papoutsoglou et al., 2019). TGF- $\beta$ signalling has been 
noticed in porcine ovarian granulosa cells after a SMAD4 knockdown study (Zhang et al., 2016). Since then, the apoptosis-inducing capacity of TGF- $\beta$ signalling was gradually proved. Some studies suggested that SMAD4 affects the FSH response because knockdown of SMAD4 significantly inhibited FSH-induced GC proliferation and estradiol production (Wang et al.). Also, SMAD4 was proved to downregulate miR143 expression by promoter binding, thus resist GC apoptosis caused by miR-143 targeting of FSHR (Xing et al., 2016). Our findings, however, implied another possible function of TGF- $\beta$ signalling during follicular atresia through the VEGFA-mediated angiogenesis and vasculogenesis processes. Although it is well known that expression of VEGFA can be regulated at the transcriptional level by several cis-acting mechanisms, its regulation by TGF-beta seems to be at the post-transcriptional level. For example, reduced stability of the VEGFA protein through TGF- $\beta$ signalling mediated ubiquitination and degradation was observed in colon carcinoma (Geng et al.). Our results provided further evidence that TGF- $\beta$ signaling could reduce VEGFA level through miRNA mediated mechanisms, which added knowledge to the functional study of TGF- $\beta$ signaling.

\section{Conclusions}

Our data provide direct evidence that miR-361-5p is up-regulated during follicular atresia and enhanced GC apoptosis by direct targeting to the 3'UTR of VEGFA mRNA and downregulating its expression. In addition, the TGF- $\beta$ signalling might play a part in VEGFA mediated GC apoptosis by transcriptional regulation of miR-361-5p expression via its common mediator SMAD4. Overall, our findings enriched the knowledge of VEGFA post-transcriptional regulation in ovarian GC apoptosis, provide novel insights into the mechanism underlying follicular atresia and ovarian physiological function studies in ovaries of mammals.

\section{Abbreviations}

TGF

transforming growth factor

VEGF

vascular endothelial growth factor

HFs

healthy follicles

AFs

atretic follicles

GC

granulosa cell

TC

theca cell

\section{Declarations}




\section{Ethics approval and consent to participate}

Animal procedures were conducted following the guidelines of the Institutional Animal Care and Use Committee of Nanjing Agricultural University.

\section{Authors' contributions}

Not applicable

\section{Availability of data and materials}

Not applicable

\section{Competing interests}

\section{The authors declare that they have no competing interests}

\section{Funding}

This work was supported by the National Natural Science Foundation of China (grant no. 31902123 and 31672421) and the Natural Science Foundation of Jiangsu Province (grant no. BK20160721).

\section{Authors' contributions}

Z.P and J.Z initiated and designed the study. M.M, X.G and J.Z performed theexperiments. M.M, X.Gand W.Yanalyzed and interpreted data;J.Z was a major contributor in writing the manuscript.All authors read and approved the final manuscript.

\section{Acknowledgements}

Not applicable

\section{References}

1. Alexander K, Jochen I, Dziunycz PJ, Adriana P, Alessia G, Hofbauer GFL, Gerber AP, Michael D. The Expression Levels of MicroRNA-361-5p and Its Target VEGFA Are Inversely Correlated in Human Cutaneous Squamous Cell Carcinoma. PLoS One. 2012;7:e49568. 
2. Berisha B, Schams D, Kosmann M, Amselgruber W, Einspanier R. Expression and localisation of vascular endothelial growth factor and basic fibroblast growth factor during the final growth of bovine ovarian follicles. J Endocrinol. 2000;167:371-82.

3. Buteaulozano H, Ancelin M, Lardeux B, Milanini J, Perrotapplanat M. Transcriptional Regulation of Vascular Endothelial Growth Factor by Estradiol and Tamoxifen in Breast Cancer Cells A Complex Interplay between Estrogen Receptors a and $\beta$. Cancer Res. 2002;62:4977.

4. Chai Z-T, Kong J, Zhu X-D, Zhang Y-Y, Lu L, Zhou J-M, Wang L-R, Zhang K-Z, Zhang Q-B, Ao J-Y. MicroRNA-26a Inhibits Angiogenesis by Down-Regulating VEGFA through the PIK3C2a/Akt/HIF-1a Pathway in Hepatocellular Carcinoma. PLoS One. 2013;8:e77957.

5. Damert A, Ikeda E, Risau W. Activator-protein-1 binding potentiates the hypoxia-induciblefactor-1mediated hypoxia-induced transcriptional activation of vascular-endothelial growth factor expression in C6 glioma cells. Biochem J. 1997;327(Pt 2):419.

6. Du X, Pan Z, Li Q, Liu H, Li Q. SMAD4 feedback regulates the canonical TGF- $\beta$ signaling pathway to control granulosa cell apoptosis. Cell Death Dis. 2018;9:151. doi:10.1038/s41419-017-0205-2.

7. Gao X, Zhang J, Pan Z, Li Q, Liu H. The distribution and expression of vascular endothelial growth factor A (VEGFA) during follicular development and atresia in the pig. Reprod Fertil Dev. 2020;32:259-66. doi:https://doi.org/10.1071/RD18508.

8. Geng L, Anathbandhu C, Geoffrey T, Jing WJLW. and S. Lu-Zhe. TGF-Beta Suppresses VEGFAMediated Angiogenesis in Colon Cancer Metastasis. PLoS One 8:e59918-.

9. Greenaway J, Connor K, Pedersen HG, Coomber BL, LaMarre J, Petrik J. Vascular endothelial growth factor and its receptor, Flk-1/KDR, are cytoprotective in the extravascular compartment of the ovarian follicle. Endocrinology. 2004;145:2896-905. doi:10.1210/en.2003-1620.

10. Hanson J, Gorman J, Reese J, Fraizer G. Regulation of Vascular Endothelial Growth Factor, VEGF, Gene Promoter by the Tumor Suppressor, WT1. Frontiers in Bioscience A. Journal Virtual Library. 2007;12:2279.

11. Jianyin Long YW, Wang W, Chang BHJ, Danesh FR. Identification of microRNA-93 as a novel regulator of vascular endothelial growth factor in hyperglycemic conditions. J Biol Chem. 2010;285:23457-65.

12. López $A O$, Olsson $F$, Llinares $A B$, Gutiérrez $H$, Latorre $R$, Candanosa $E$, Guillénmartínez A, Izquierdorico MJ. Expression of the vascular endothelial growth factor system (VEGF) in the porcine oviduct during the estrous cycle. Theriogenology. 2017;93:46-54.

13. Lin F, Li R, Pan ZX, Zhou B, Yu DB, Wang XG, Ma XS, Han J, Shen M, Liu HL. miR-26b promotes granulosa cell apoptosis by targeting ATM during follicular atresia in porcine ovary. PLoS One. 2012;7:e38640.

14. Liu B, Lu B, Wang X, Jiang H, Kuang W. 2019. MiR-361-5p inhibits cell proliferation and induces cell apoptosis in retinoblastoma by negatively regulating CLDN8. Child s Nervous System 35.

15. Liu J, Li X, Yao Y, Li Q, Pan Z, Li Q. miR-1275 controls granulosa cell apoptosis and estradiol synthesis by impairing LRH-1/CYP19A1 axis. Biochim Biophys Acta. 2018;1861:246. 
16. Ma F, Song H, Guo B, Zhang Y, Zheng Y, Lin C, Wu Y, Guan G, Sha R, Zhou Q, Wang D, Zhou X, Li J, Qiu $X$. MiR-361-5p inhibits colorectal and gastric cancer growth and metastasis by targeting staphylococcal nuclease domain containing-1. Oncotarget. 2015;6:17404-16. doi:10.18632/oncotarget.3744.

17. Ma F, Zhang L, Ma L, Zhang Y, Zhang J, Guo B. MiR-361-5p inhibits glycolytic metabolism, proliferation and invasion of breast cancer by targeting FGFR1 and MMP-1. J. Exp. Clin. Cancer Res. $36: 158$.

18. Manabe N, Goto Y, Matsudaminehata F, Inoue N, Maeda A, Sakamaki K, Miyano T. Regulation mechanism of selective atresia in porcine follicles: regulation of granulosa cell apoptosis during atresia. Journal of Reproduction Development. 2004;50:493-514. doi:10.1262/jrd.50.493.

19. Marchal R, Vigneron C, Perreau C, Balipapp A, Mermillod P. Effect of follicular size on meiotic and developmental competence of porcine oocytes. Theriogenology. 2002;57:1523. doi:10.1016/S0093691X(02)00655-6.

20. Meister G, Landthaler M, Patkaniowska A, Dorsett Y, Teng G, Tuschl T. Human Argonaute2 mediates RNA cleavage targeted by miRNAs and siRNAs. Mol Cell. 2004;15:185-97. doi:10.1016/j.molcel.2004.07.007.

21. Papoutsoglou P, Louis C, Coulouarn C. 2019. Transforming Growth Factor-Beta (TGFbeta) Signaling Pathway in Cholangiocarcinoma. 8doi:10.3390/cells8090960.

22. Shimizu T, Jiang JY, Sasada H, Sato E. Changes of messenger RNA expression of angiogenic factors and related receptors during follicular development in gilts. Biol Reprod. 2002;67:1846-52.

23. Stouffer RL, Martínezchequer JC, Molskness TA, Xu F, Hazzard TM. Regulation and action of angiogenic factors in the primate ovary. Arch Med Res. 2001;32:567-75.

24. Wahid F, Shehzad A, Khan T, Kim YY. MicroRNAs: synthesis, mechanism, function, and recent clinical trials. Biochim Biophys Acta. 2010;1803:1231-43. doi:10.1016/j.bbamcr.2010.06.013.

25. Wang W, Chen X, Li X, Wang L, Zhang H, He Y, Wang J, Zhao Y, Zhang B, Xu Y. Interference RNAbased silencing of endogenous SMAD4 in porcine granulosa cells resulted in decreased FSHmediated granulosa cells proliferation and steroidogenesis. Reproduction 141:643-651.

26. Xing D, Zhang L, Li X, Pan Z, Liu H, Li Q. TGF- $\beta$ signaling controls FSHR signaling-reduced ovarian granulosa cell apoptosis through the SMAD4/miR-143 axis. Cell Death Dis. 2016;7:e2476.

27. Yamamoto S, Konishi I, Tsuruta Y, Nanbu K, Mandai M, Kuroda H, Matsushita K, Hamid AA, Yura Y, Mori T. Expression of vascular endothelial growth factor (VEGF) during folliculogenesis and corpus luteum formation in the human ovary. Gynecological Endocrinology the Official Journal of the International Society of Gynecological Endocrinology. 1997;11:371-81.

28. Zhang HY, Wang W, He Y, Wang L, Tian KX, Song XG, Xu YX. Effects of Silencing Smad4 Gene by Small Interfering RNA on Apoptosis of Porcine Granulosa Cells. Chinese Journal of Cell Biology. 2010;32:596-600.

29. Zhang J, Liu Y, Yao W, Li Q, Liu HL, Pan Z. Initiation of follicular atresia: gene networks during early atresia in pig ovaries. Reproduction. 2018a;156:23-33. 
30. Zhang J, Xu Y, Liu H, Pan Z. MicroRNAs in ovarian follicular atresia and granulosa cell apoptosis. Reprod Biol Endocrinol. 2019;17:9.

31. Zhang L, Du X, Wei S, Li D, Li Q. A comprehensive transcriptomic view on the role of SMAD4 gene by RNAi-mediated knockdown in porcine follicular granulosa cells. Reproduction. 2016;152:81-9. doi:10.1530/rep-16-0034.

32. Zhang L, Lv Z, Xu J, Chen C, Ge Q, Li P, Wei D, Wu Z, Sun X. 2018b. MicroRNA-134 inhibits osteosarcoma angiogenesis and proliferation by targeting the VEGFA/VEGFR1 pathway. FEBS J.

33. Zhao WJ, Zhang HF, Su JY. Downregulation of microRNA-195 promotes angiogenesis induced by cerebral infarction via targeting VEGFA. Mol Med Report. 2017;16:5434-40.

34. Zhou B, Ma R, Si W, Li S, Xu Y, Tu X, Wang Q. MicroRNA-503 targets FGF2 and VEGFA and inhibits tumor angiogenesis and growth. Cancer Lett. 2013;333:159-69.

35. Zhu X, Er K, Mao C, Yan Q, Xu H, Zhang Y, Zhu J, Cui F, Zhao W, Shi H. miR-203 suppresses tumor growth and angiogenesis by targeting VEGFA in cervical cancer. Cell Physiol Biochem. 2013;32:6473.

\section{Figures}



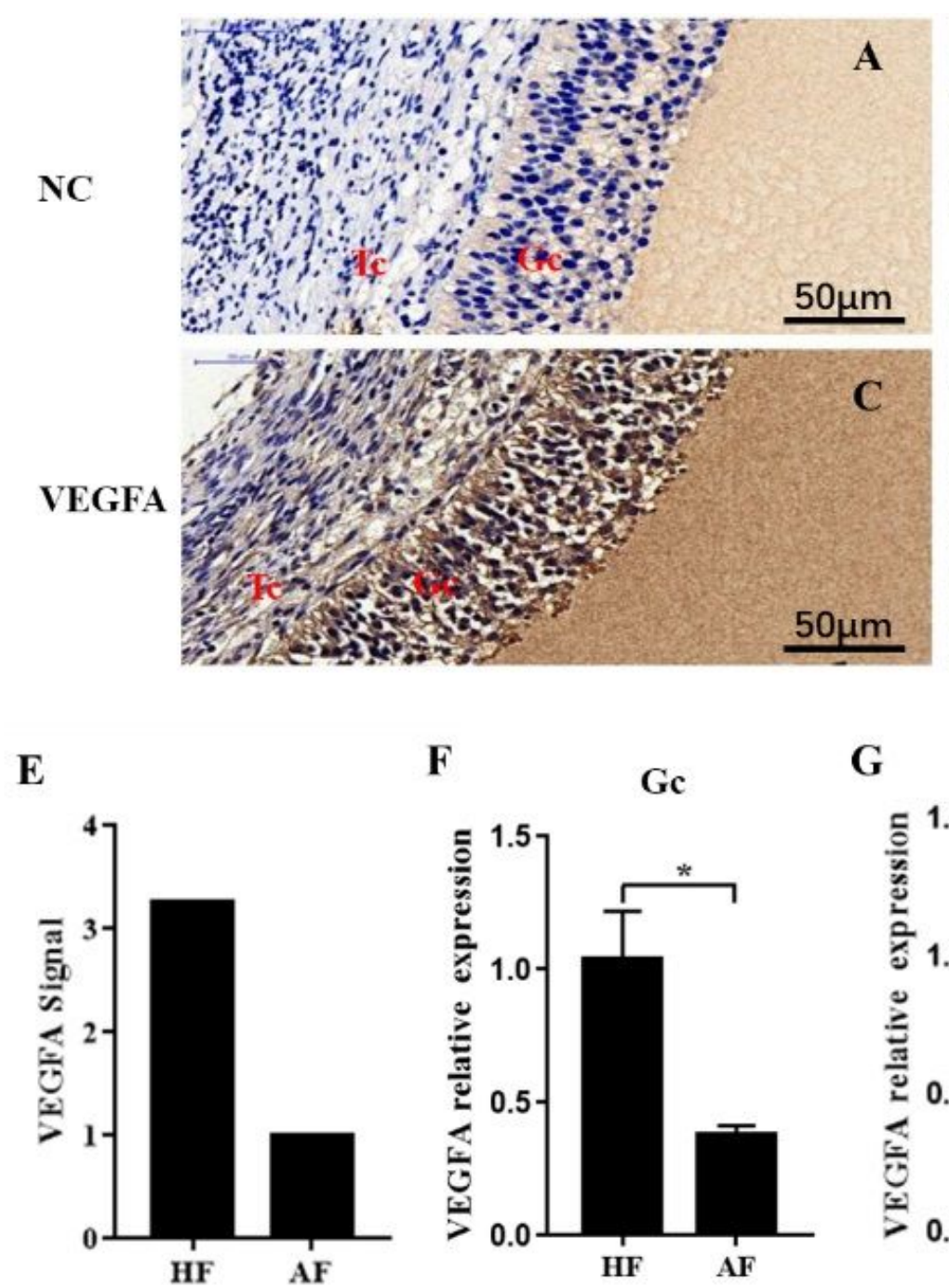

F

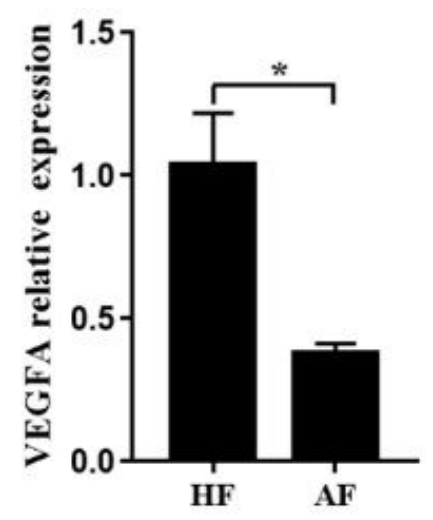

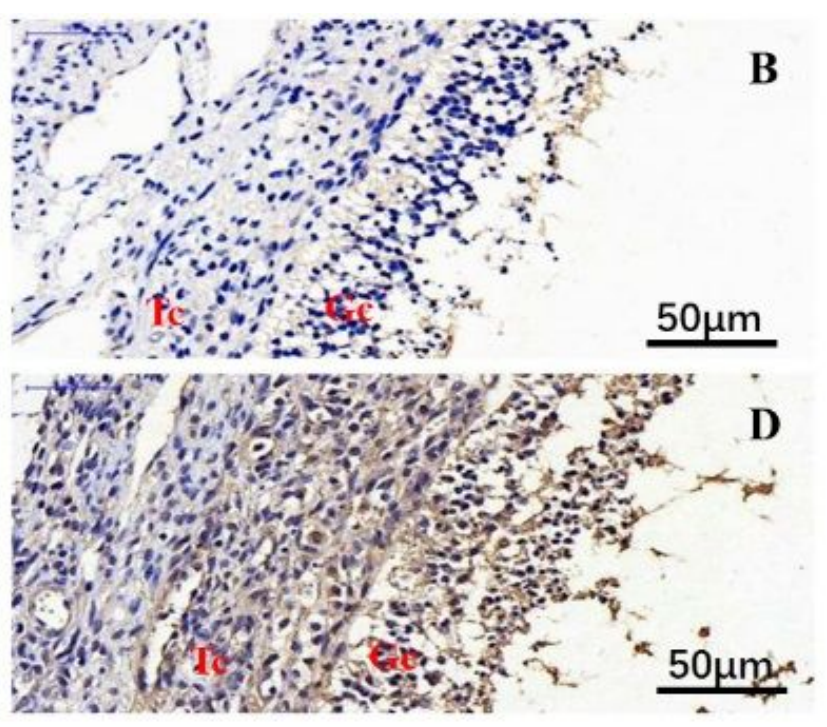

G

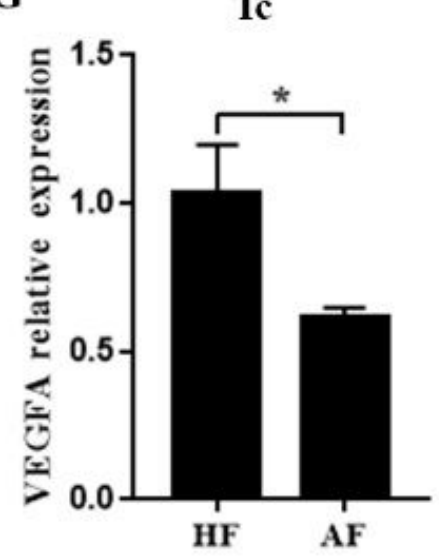

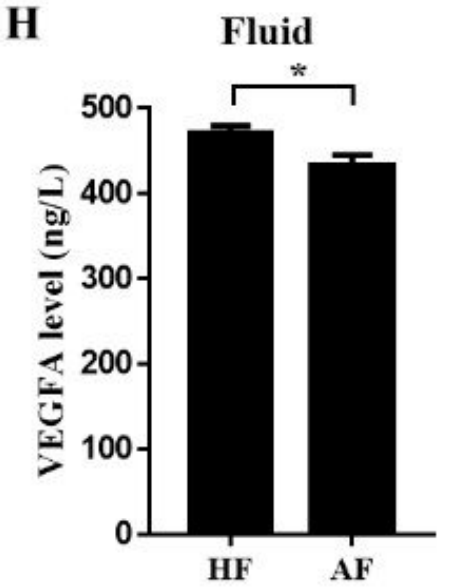

Figure 1

Expression of VEGFA in healthy and atretic antral follicles. A-D: Immunolocalization of VEGFA in healthy $(A, C)$ and atretic $(B, D)$ antral follicles; E: The signalintensity of VEGFA in follicles detected by GeneChip Porcine GenomeArray; F, G: relative expression levels of VEGFA in GC and TC respectively detected by qRTPCR; $\mathrm{H}$ : expression level of VEGFA in follicle fluid detected by ELISA. NC, negative control; HF, healthy follicle; $A F$, atretic follicle; $G C$, granulosa cell; Tc, theca cell; scale bar $=50 \mu \mathrm{m}$. Data are expressed as the mean \pm SEM. ${ }^{*} p<0.05$. 
DAPI
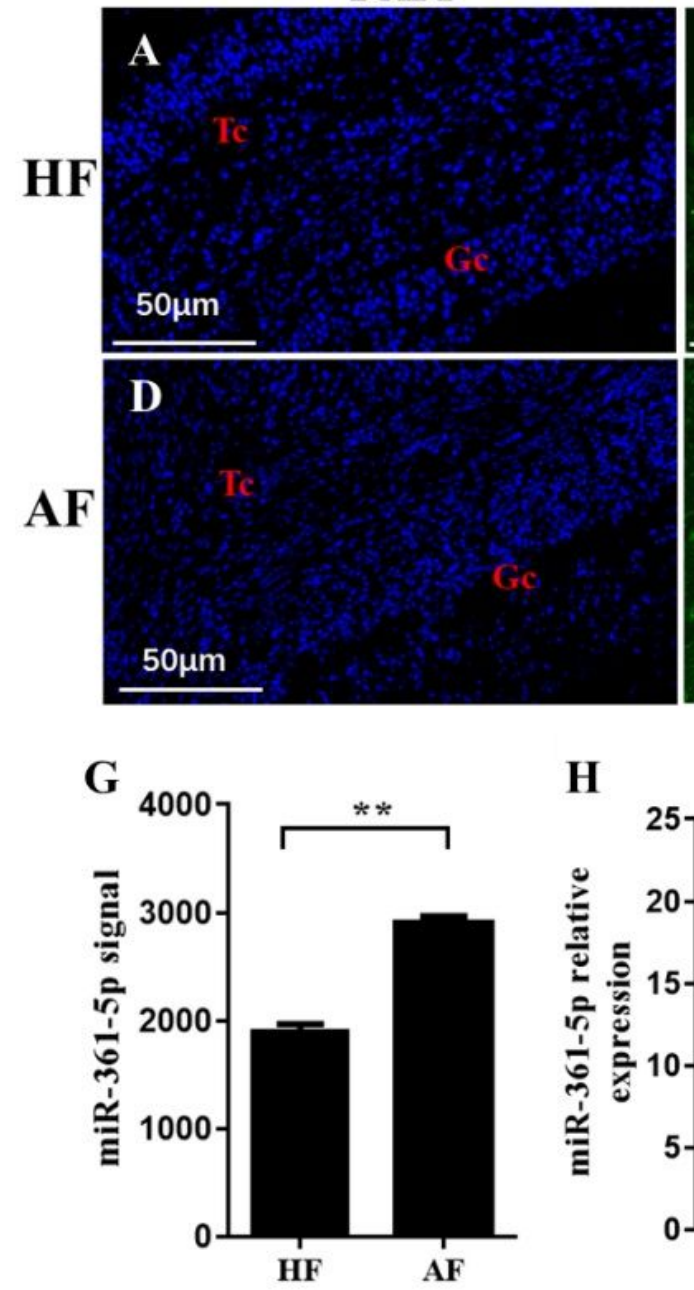

mir-361-5p
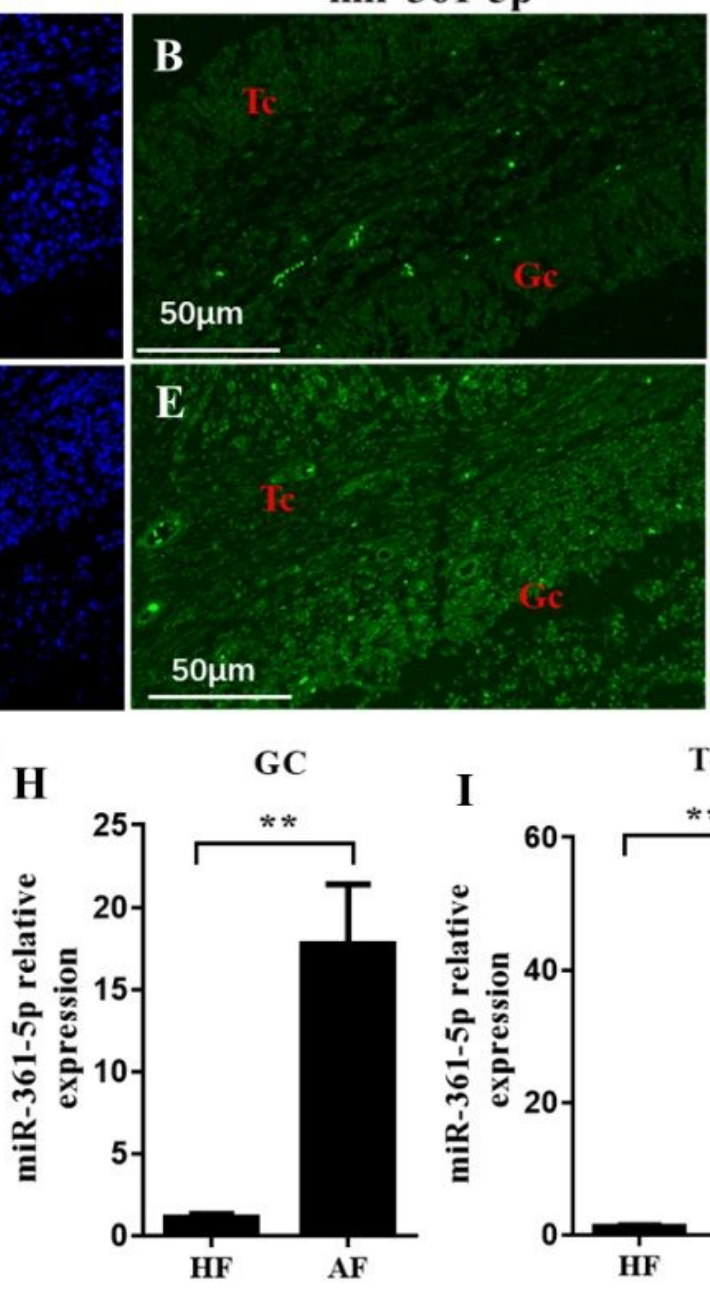

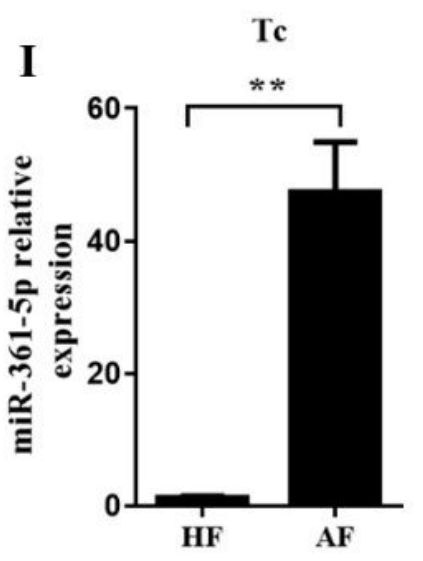

Merge

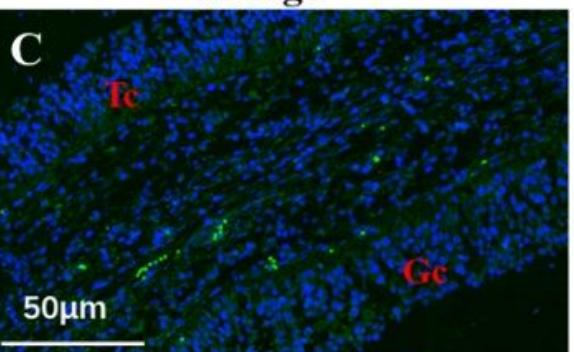

F

$50 \mu \mathrm{m}$

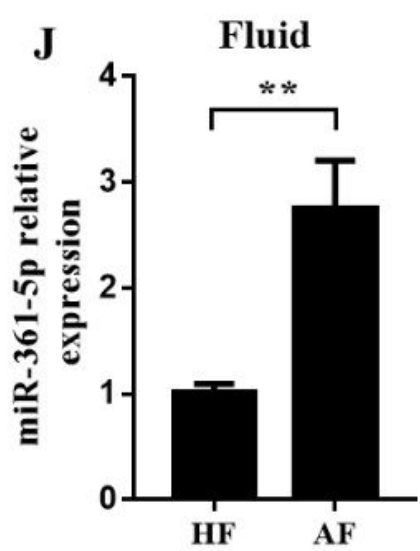

Figure 2

Expression of mir-361-5p in healthy and atretic antral follicles. A-F: RNA-FISH was utilized to examine the localization of mir-361-5p in healthy and atretic antral follicles; G: Signalintensity of mir-361-5p in follicles detected by $\mu$ Paraflo ${ }^{\text {TM }}$ microfluidic chip; $\mathrm{H}-\mathrm{J}$ : relative expression levels of mir-361-5p in GC, TC, and follicle fluid respectively detected by qRT-PCR. HF, healthy follicle; AF, atretic follicle; GC, granulosa cell; TC, theca cell; scale bar $=50 \mu \mathrm{m}$. Data are expressed as the mean \pm SEM. ${ }^{*} \mathrm{p} p<0.01$. 
A

mir-361-5p $3^{\prime} \ldots$ CAUGgGgacCUCUAAGACUAUU... $5^{\prime}$ VEGEA-3' UTR-WT $5^{\prime} \ldots$ TGTATATATGTGATTCTGATA.... $3^{\prime}$ VEGEA-3' UTR-Mut 5 '... TGTATATATGTGATTCTGATA.... $3^{\prime}$

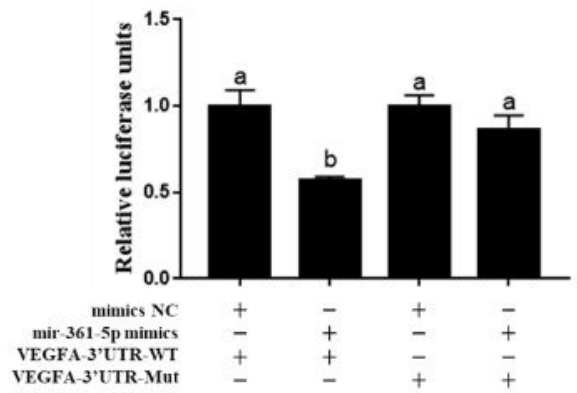

B

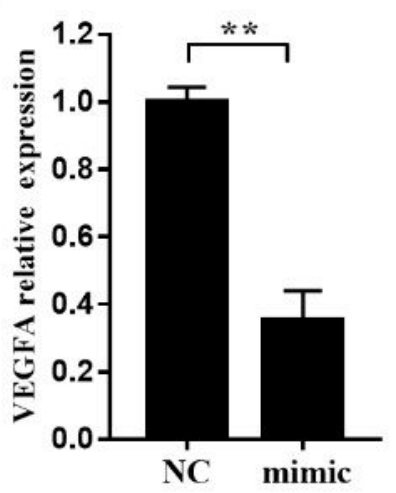

C

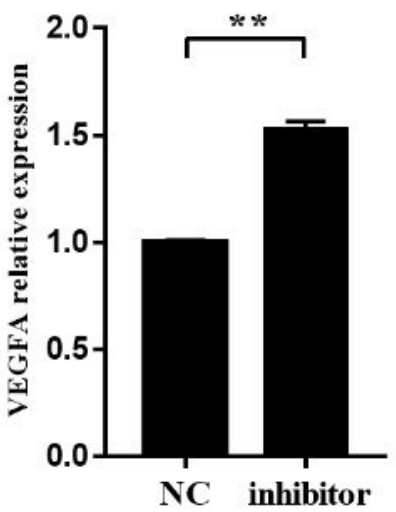

D

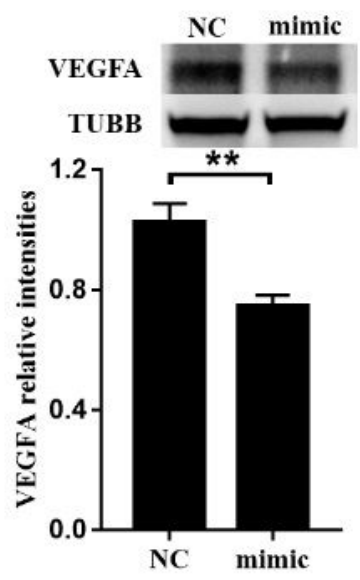

E
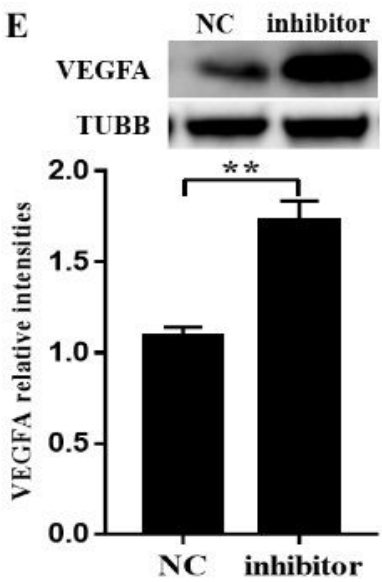

F

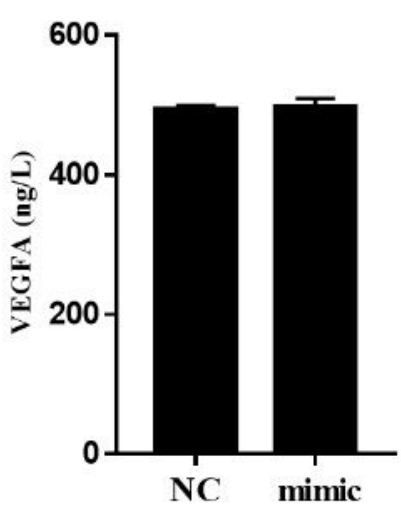

G

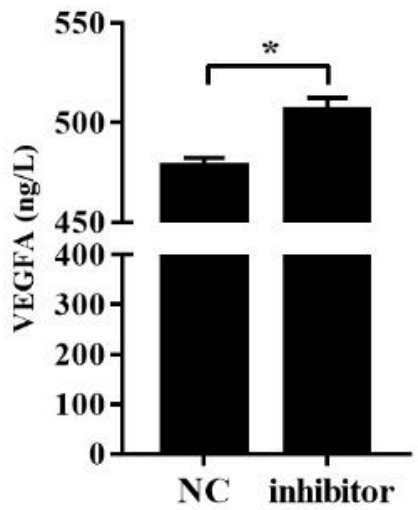

\section{Figure 3}

Negative regulation of VEGFA by miR-361-5p through direct 3'UTR binding. A: The direct targeting of VEGFA by miR-361-5p confirmed by luciferase reporter assay in 293 cells; B,C: The mRNA levels of VEGFA after transfection of miR-361-5p mimics or inhibitors in porcine GCs; D,E: The protein levels of VEGFA after transfection of miR-361-5p mimics or inhibitor in porcine GCs; F,G: The levels of VEGFA after transfection of miR-361-5p mimics and inhibitors in culture media. $n=3$ cell culture wells per group. Data are expressed as the mean \pm SEM. Significant differences $(p<0.05)$ are indicated by different letters or * $p$ $<0.05,{ }^{*} \mathrm{p}<0.01$ 
A
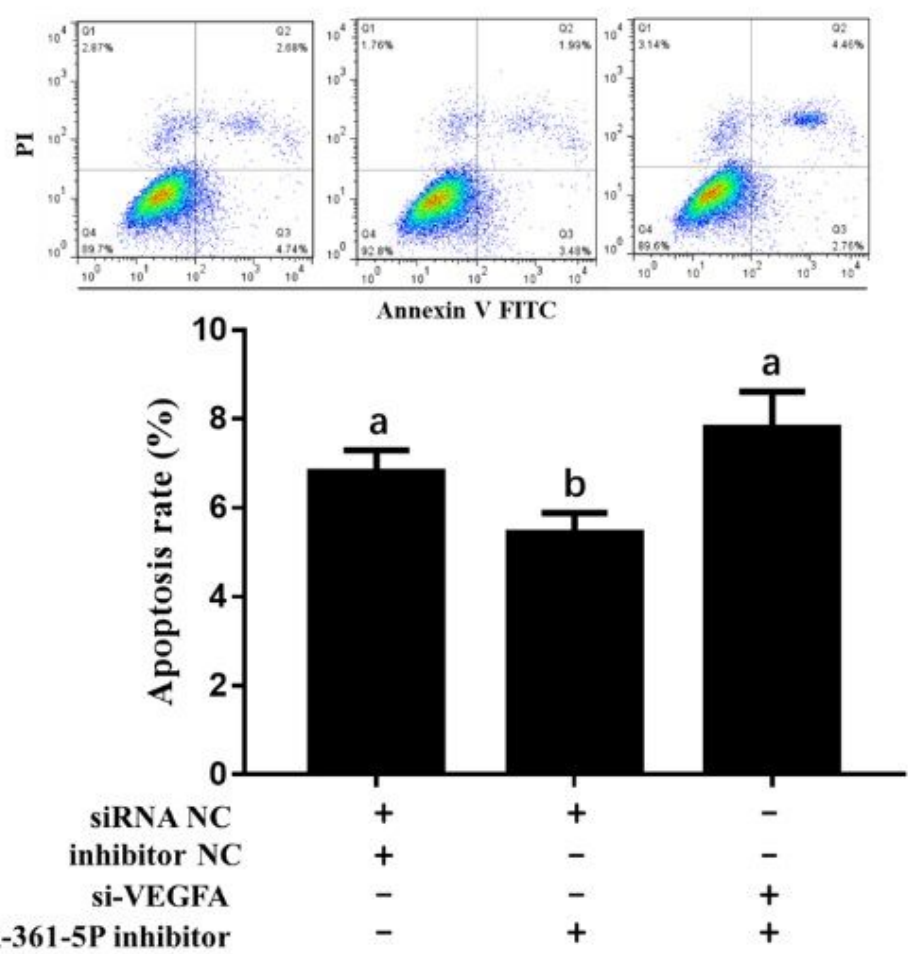

B c-CAS3 TUBB

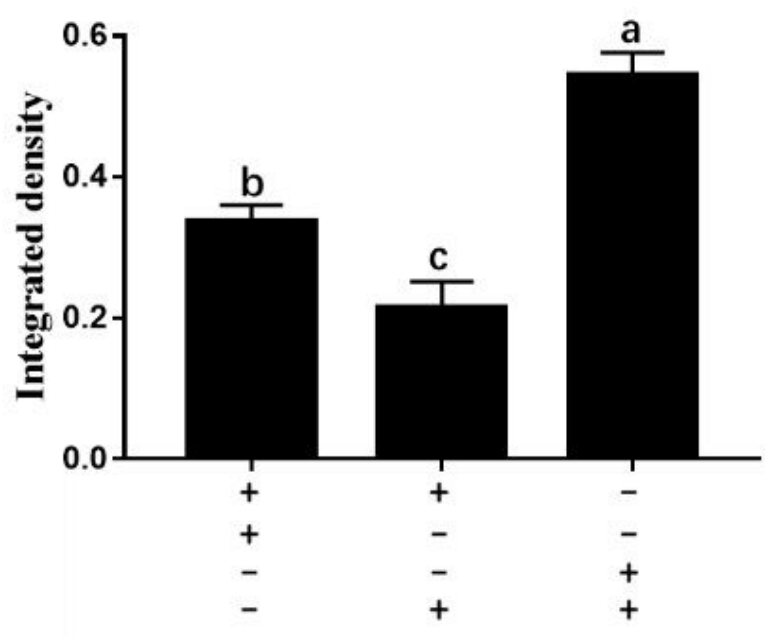

\section{Figure 4}

miR-361-5p regulates GC apoptosis through VEGFA. A: GC apoptosis rate decreased after the transfection of miR-361-5p inhibitor and reversed after the addition of VEGFA siRNA detected by FACS analysis. B: The protein levels of cleaved caspase 3 (c-CAS3) was down-regulated after the transfection of miR-361-5p inhibitor and reversed after the addition of VEGFA siRNA.Data are expressed as the mean \pm SEM. Significant differences $(p<0.05)$ are indicated by different letters. 


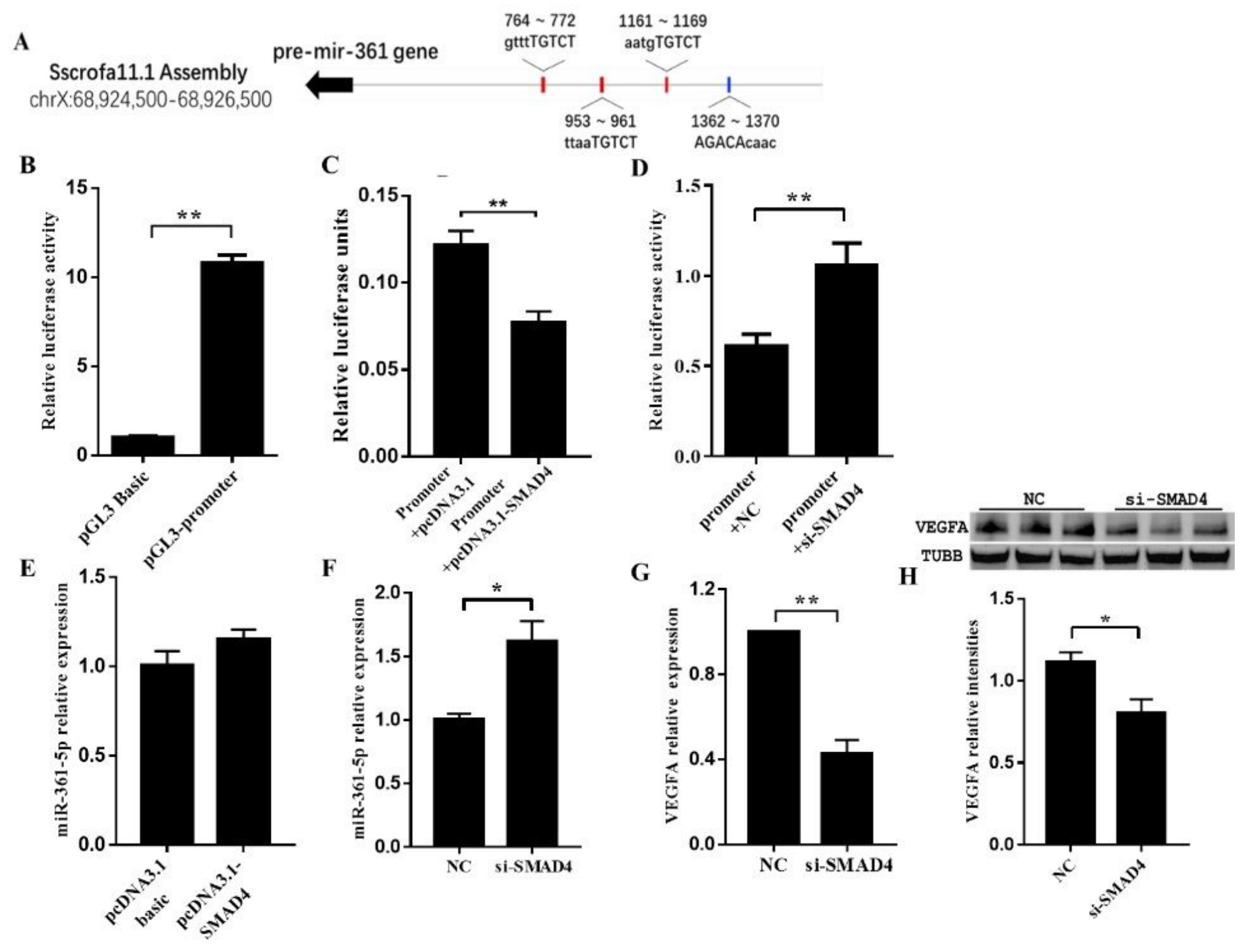

\section{Figure 5}

Transcription factor SMAD4 was involved in miR-361-5p mediated VEGFA expression. A: Schematic diagram showing the genome location of the miR-361-5p coding gene and potential SMAD4 binding sites. B: The promoter activity ofMIR361upstream region confirmed by dual-luciferase reporter assay; C,D: Overexpression and knockdown of SMAD4 weaken and enhanced MIR361 promoter activity respectively; $\mathrm{E}$ : The expression of miR-361-5p was not affected after SMAD4 overexpression; $F$ : The expression of miR361-5p was up-regulated after SMAD4 knockdown; G,H: The expression of VEGFA mRNA and protein levels was down-regulated after SMAD4 knockdown. $\mathrm{n}=3$ cell culture wells per group. Data are expressed as the mean \pm SEM. ${ }^{*} p<0.05,{ }^{* *} p<0.01$. 


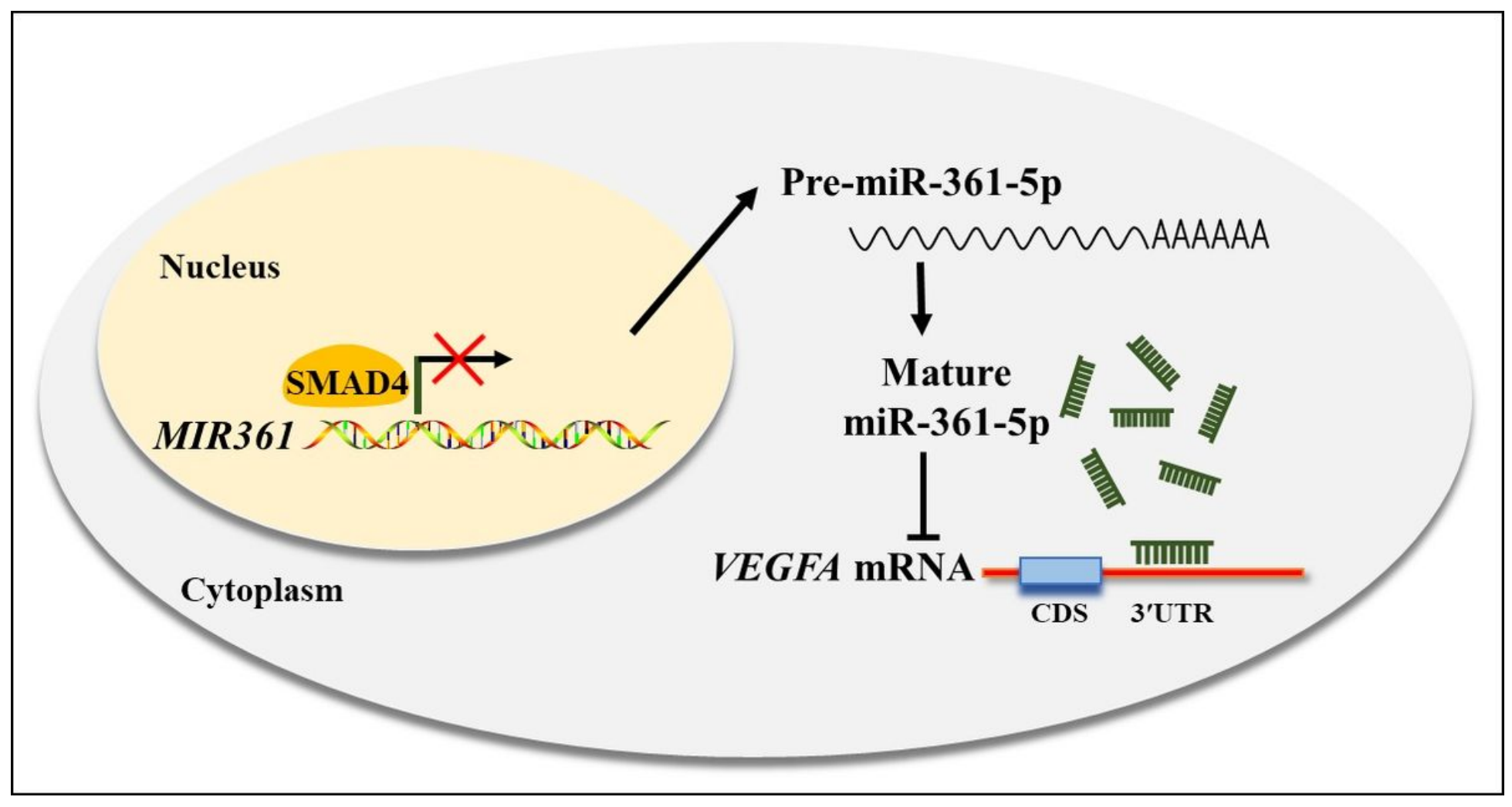

Figure 6

Schematic diagram of SMAD4/ miR-361-5p/VEGFA regulatory signalling in porcine GCs. SMAD4 negatively regulates MIR361 transcription by binding to MIR361 promoter, while matured miR-361-5p reduces VEGFA expression level by directly bind to 3'UTR VEGFA mRNA.

\section{Supplementary Files}

This is a list of supplementary files associated with this preprint. Click to download.

- Tables13.doc 